\title{
Aesthetic Treatment of Traumatized Anterior Teeth: A Case Report
}

\author{
Nathania Pramudita ${ }^{1, *}$ Fransisca Debby Rosalia ${ }^{2,}$ Yulita Kristanti ${ }^{3,}$ Diatri Nari Ratih ${ }^{4}$ \\ ${ }^{1}$ Conservative Dentistry Specialist Program, Faculty of Dentistry, Universitas Gadjah Mada, Yogyakarta, Indonesia \\ ${ }^{2}$ Conservative Dentistry Specialist Program, Faculty of Dentistry, Universitas Gadjah Mada, Yogyakarta, Indonesia \\ ${ }^{3}$ Departement of Conservative Dentistry, Faculty of Dentistry, Universitas Gadjah Mada, Yogyakarta, Indonesia \\ ${ }^{4}$ Departement of Conservative Dentistry, Faculty of Dentistry, Universitas Gadjah Mada, Yogyakarta, Indonesia \\ ${ }^{*}$ Corresponding author. Email: diatri@.ugm.ac.id
}

\begin{abstract}
The emergency of a traumatically injured oral and maxillofacial region occurs frequently, and such injuries certainly need dental treatment. Therapy of traumatic dental injury aims to restore the function of the tooth; Towards this goal dentists will need a carefully managed treatment planning that often involves a multidisciplinary approach. This case report aims to present the treatment of traumatized anterior teeth aesthetically. Twenty year old male patient came to Conservative Dentistry clinic of Universitas Gadjah Mada Dental Hospital, complaining about the traumatized crown fractures of front teeth 12, 11, 21 and 22 caused by an injury in the previous 4 months. Clinical examination revealed a crown fracture with exposed pulp chamber on all maxillary incisors with grade 1 mobility. Radiographic examination showed a radiolucent area in the periapical region of right maxillary lateral incisor and left maxillary central incisor. Root canal treatments were performed on all maxillary incisors. Crown lengthening without bone reduction was carried out after root canal treatment, then it was followed by placing of a customized fiber-reinforced post and all-porcelain crown. After the treatment, the comprehensive traumatic injury of the anterior teeth can be restored aesthetically, by way of a combined treatment using endodontic and periodontic treatment.
\end{abstract}

Keywords: Aesthetic treatment, traumatic injury, root canal treatment, crown lengthening

\section{INTRODUCTION}

Dental trauma is one of the most prevalent clinical problems in children, adolescents and adults. Maxillary incisors are the most frequently affected teeth in both primary and permanent dentition injuries [1]. thus, esthetic and functional results must be considered when determining the proper treatment method [2].

It has been advocated that teeth become more fragile after endodontic treatments, and this increases the failure rate of post-endodontics restorations due to loss of tooth structure, moisture, and flexibility of the dentine. Therefore, extensive tooth loss after cavity preparation represents a real challenge in the restoration of endodontically treated teeth [3]. When the incisors are decayed or fractured, the situation is complicated as it needs prior reconstruction. Currently, fiber posts and cores solve the problem thanks to their elastic behaviour [2]. Recently, several different posts and types of core materials are in use, whereby fiber-reinforced post (FRC) have been used with acceptable results both in clinical practise and in research. Fiber-reinforced post has shown a superior performance as compared to metal post due to its "dentin-like" elastic modulus and exceptional aesthetic properties [3].

The restoration of endodontically treated tooth might be complicated in cases with an inadequate height of the remaining coronal wall. This clinical scenario could result in a loss of the ferrule, which may significantly jeopardize the structural integrity of the tooth. Therefore, to provide an adequate ferrule, a crown lengthening procedure may be indicated before the restoration of the endodontically treated tooth [4]. The crown lengthening procedures are routine in the clinical practice. The exposure of tooth surface by apically displacing the gingival tissue and the bone margins facilitates placement of restorative margins on the sound tooth surface. In doing so, the procedure enables maintaining biological width of 2-3 $\mathrm{mm}$ that ensures health of the periodontal tissues [5].

This report presents a case of traumatic injury on maxillary incisors that was treated with a combination between endodontic and periodontic treatment. The aim of this report is to present the aesthetic treatment of anterior teeth due to traumatic injury.

\section{CASE REPORT}

A 20-year-old male patient came to Conservative Dentistry clinic of Universitas Gadjah Mada Dental Hospital and complained about his fractured upper front teeth that was caused by a trauma in the previous four months. The patient revealed that the teeth were restored by a dentist one year ago due to large cavities. Two weeks after injury, the patient felt some pain on his fractured anterior teeth but it was gradually relieved. The patient had a high preference on sour and sweet 
tasting food. Overall, the patient had a good health with an insignificant systemic medical history.

Intraoral examination showed that there were cavities with pulp involvement on teeth $12,11,21,22$, and pulp vitality test was negative on all maxillary incisors, with grade 1 mobility. The patient's OHI-S score was 2, and saliva examination revealed that he had a low $\mathrm{pH}$ level. Intraoral and panoramic radiographic examination showed a radiolucent area in the periapical region of right maxillary lateral incisor and left maxillary central incisor. Clinical and radiographic examination revealed Ellis class IV fracture, nonvital pulp with asymptomatic apical periodontitis on maxillary central and lateral incisors.

Gingival margin on anterior maxillary region was firm, red, and unstippled. Interdental papillae had lost its knifelike shape and displays puffy. The remaining of coronal wall on tooth $12,11,21$, and 22 was inadequate. This might result in loss of ferrule. Therefore, a crown lengthening procedure may be indicated before the restoration of the teeth.

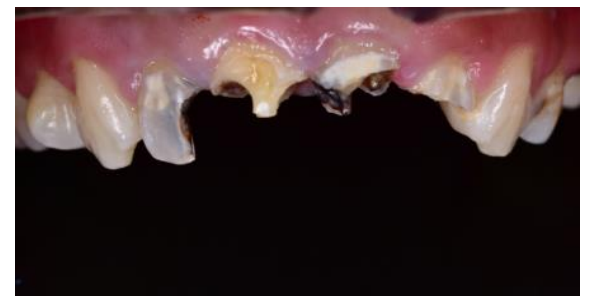

Figure 1 Clinical presentation of teeth prior to treatment

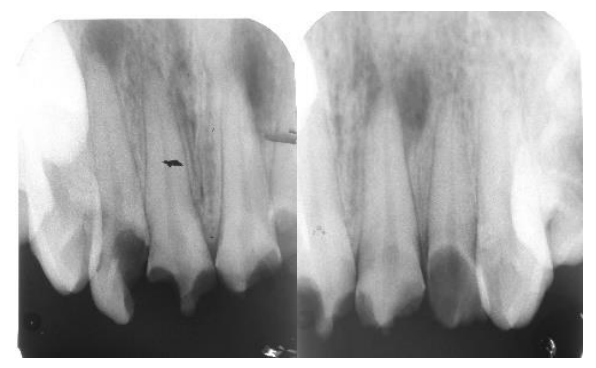

Figure 2 Radiographic examination showed radiolucent area on periapical of tooth 12 and 21

Treatment planning for this case consisted of providing information to patient about his condition, followed by endodontic treatment, crown lengthening, application of customized fiber reinforced post, and restoration of maxillary incisors with all porcelain crown.

\section{CASE MANAGEMENT}

Face analysis, dentofacial analysis, dental analysis, gingival analysis and space analysis were done to establish treatment planning of the maxillary incisors. Restoration using the available space was done with selective grinding in order to obtain aesthetic aspect of the teeth, and to make the teeth appear less wide and to boost the masculinity character of the patient. The patient signed an informed consent before the procedure was performed.
Endodontic treatment was done on tooth 12, 11, 21, and 22. Maxillary incisors were isolated using rubber dam to prevent contamination. An access cavity was prepared and extirpation was performed using barbed broaches. After the working length of determination on each maxillary incisors was established, biomechanical preparation was carried out with crown down technique using Protaper Gold up to \#F4. The root canal was irrigated with $2,5 \% \mathrm{NaOCl}$ solution for every file change. The root canal was then irrigated with $2,5 \%$ $\mathrm{NaOCl}, 17 \%$ EDTA, $2 \%$ Chlorhexidine, and normal saline every time the solution was changed. The canal was dried with paper point and dressing material, $\mathrm{CaOH}$, was applied inside root canal, followed by temporary restoration. Obturation was done a week later with single cone technique, using F4 gutta percha and epoxy resin -based sealer. The cavities were restored with glass ionomer cement and temporary restoration.
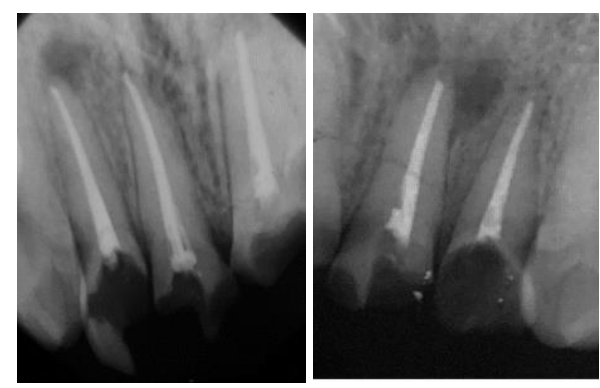

Figure 3 Radiographic examination on tooth 12, 11, 21, 22 after endodontic treatment was done

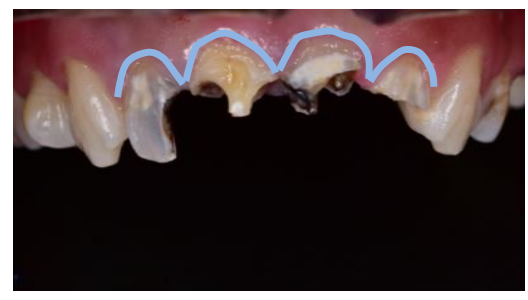

Figure 4 Ginival area that incised (blue line)

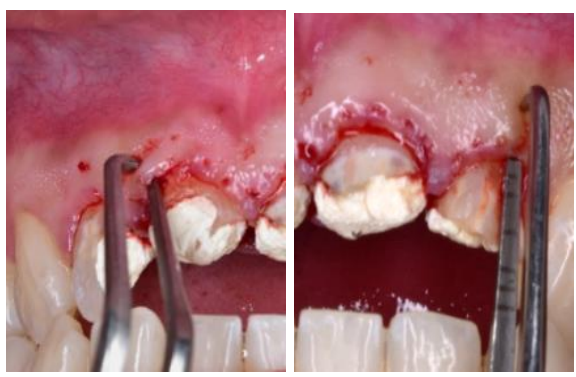

Figure 5 The line of gingival incision was marked using socket marker 


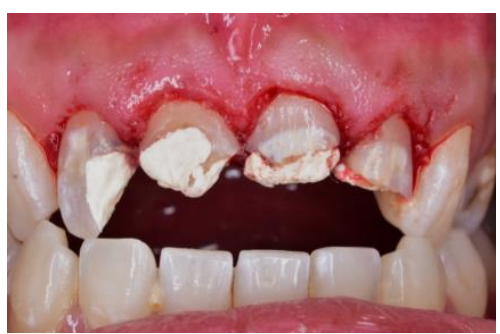

Figure 6 The result of crown lengthening procedure

The crown lengthening procedure was performed the following week. This procedure was done on gingival margin of $12,11,21$, and 22 in order to get adequate coronal wall. Periodontal probing on labial pocket revealed that sulcus depth was $2 \mathrm{~mm}$. Radiographic examination showed that range of cementoenamel junction to alveolar crest was 0,5 $\mathrm{mm}$. Reduction of gingival margin that needed was $1,5 \mathrm{~mm}$, meanwhile the biologic width was $2,04 \mathrm{~mm}$. Thus, the estimated range of crown lengthening procedure was 1,04 $\mathrm{mm}$. bone reduction was not done in this procedure due to 2 $\mathrm{mm}$ of sulcus depth $(>1,04 \mathrm{~mm})$.

The patient was asked to sign an informed consent prior to the surgery. The working area was disinfected with iod, then anesthetized. Labial aspect of the gingiva was marked using periodontal marker as a guideline for incision. Incision was done using Kirkland and Orban. Then the gingiva was irrigated with normal saline and light pressure was applied to minimize bleeding. Periodontal pack was placed on surgery area. The patient was given a prescription drugs and postsurgery instruction. Follow up of the crown lengthening procedure was done a week later. The patient did not have any complain after the surgery. Clinical examination showed that the periodontal pack was still on its place, and healing of the gingival tissue was normal.

Preparation on the hard tissue of the teeth in order to place fiber post was done on the same day. Finishing line of chamfer was placed on $0,5 \mathrm{~mm}$ of subgingival on the labial aspect. The preparation was polished using superfine finishing diamond bur. Preparation of root canal for custom fiber post was done using peso reamer. Then, the individual fiber post (Everstick Post, GC) was inserted into the prepared canal and light was cured for 20 seconds. The fiber was then removed from the root canal. Etching agent was applied on fiber post and cavity for 15 seconds and rinsed with water, then it was dried with paper point. The root canal and cavity were smeared with bonding agent and light cured for 20 seconds. Self-adhesive resin cement was placed into root canal and the fiber post was inserted. Excess of resin cement was removed before setting. The remaining of the fiber post was made into core with self-curing multicore core built-up composite (Multicore, Ivovlar, Vivadent).

The preparation, finishing, and polishing of the core were done for fabricating the all porcelain crown. The shade of the teeth was determined using Ivoclar and it obtained D2 shade. Double impression using hydrophilic polysiloxane impression material (Exaflex, GC) and vinyl polysiloxane impression material (Putty, GC) was done for maxillary dentition, meanwhile mandibular dentition was impressed using irreversible hydrocolloid (Alginat, GC). Temporary crown was made using light cured resin and placed using zinc phosphate cement.

The all porcelain crown was inserted on the next visit using self-adhesive resin cement shade A2. Resin excess was removed before it was set and light cured for 20 seconds from each labial and palatal aspect. Correction of the occlution was done using articulating paper and the interdental was cleaned using dental floss. Polishing was done using polishing porcelain bur (EVE Diacomp).

Follow up after one week showed that patient was satisfied with the result and did not have any complain. Clinical examination showed an insignificant result and the patient was given an instruction to maintain his oral hygiene and have his teeth check with the dentist regularly every 6 months.

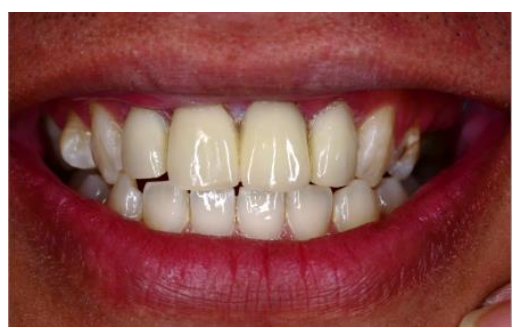

Figure 7 Clinical appearance after insertion of all porcelain crown

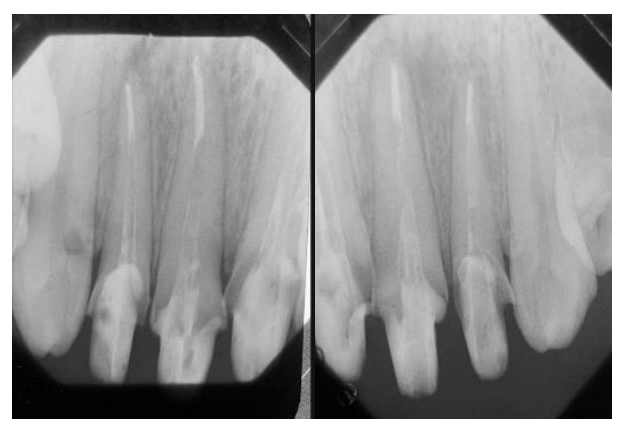

Figure 8 Periapical radiographic picture after the treatment was done

\section{DISCUSSION}

The treatment performed and presented in this clinical case report is one of the many possible options that could have been used to restore the condition of this patient. Selection of the treatment plan should be made considering the desired advantage and disadvantage and limitation of the patient.

In such occasions the dentist should weight the clinical findings and patients' concerns in the balance to determine if the tooth or teeth should be extracted (and replaced by dental implants) or be restored. Although implants have reasonably high success rates, implant failures must be considered before establishing treatment plan [6]. 
Generally, dental trauma can involve pulp both directly and indirectly, so endodontics is considered to play an important role to evaluate and treat dental injuries. Fractures that involve the edge of gingiva area need an endodontic treatment followed with crown restoration using post and core. Extensive tooth loss after cavity preparation represents a real challenge in the restoration of endodontically treated teeth. Thus, in such a specific clinical scenario, the use of intra-canal posts may be indicated to increase the retention of the core and/or of the coronal restoration. The main function of a post and core is to provide stability to a restored tooth and to protect tooth by evenly dispersing and distributing masticatory forces along the tooth. In this case, all of the maxillary incisors were impaired so that the aesthetic aspect must be considered. Fiber-reinforced posts were used in this case because fiber-reinforced post has shown superior performance as compared to metal post due to its "dentinlike" elastic modulus and exceptional aesthetic properties $[3,7]$.

Caries and fracture lines on teeth extending apical to the gingival margins, teeth with altered passive eruption, longer crown length to enhance aesthetic outcomes and correction of supra-erupted teeth are primary reasons to require crown lengthening procedure [5]. The fracture resistance and prosthetic longetivity of endodontically treated tooth have been associated with the presence of a ferrule. A ferrule is defined as band or ring of restorative material surrounding the crown or root of a tooth to provide strength. It has been reported that teeth without a ferrule show the highest values of variation of success/survival compared with endodontically treated tooth with an adequate ferrule.[4]

The restoration of endodontically treated tooth might be complicated in cases with an inadequate height of the remaining coronal wall. This clinical scenario could result in a loss of the ferrule, which may significantly jeopardize the structural integrity of the tooth. Therefore, to provide an adequate ferrule, a crown lengthening procedure may be indicated before the restoration of the endodontically treated tooth [4].

Functional crown lengthening procedure aims to expose the surface of root by placing the gingival tissue and alveolar crest apically. This procedure applied to recover the biologic width that provide sufficient retention and resistance for restoration [8]. Final restoration post re-treatment of root canal should be able to protect and maintain the remaining tooth structure and restore mastication and aesthetic function. This final restoration is one of the factors that influence the success of retreatment [9].

A short clinical crown cannot be evaluated by visual inspection alone. Hence, a comprehensive examination that includes clinical examination, radiographic examination and diagnostic cast analysis is essential for successful rehabilitation. The biologic width is defined as the dimension of the soft tissue, which is attached to the portion of the tooth coronal to the crest of alveolar bone. This term was based on the work of Gargiulo et.al. who established that there is a definite proportional relationship between the alveolar crest, the connective tissue attachment, the epithelial attachment, and the sulcus depth. Based on this work, the biologic width is routinely stated to be $2,04 \mathrm{~mm}$, which represents the sum of the epithelial and connective tissue measurements [6]. In this case, periodontal probing on labial pocket revealed that sulcus depth was $2 \mathrm{~mm}$, meanwhile the range of crown lengthening was $1,04 \mathrm{~mm}$. Therefore, the bone reduction procedure was not done in this procedure.

A short clinical crown that found in this case is one of indications of functional crown lengthening in order to maintain the biological width. Moreover, functional crown lengthening also provide supragingival structure with $2 \mathrm{~mm}$ height or more in tooth with subgingival cavity, thus can offer the ferrule effect that enhance resistance of the restoration. Jovanovski et.al. reveal that restored tooth without ferrule effect tends to undergo fracture compared with tooth with 2 $\mathrm{mm}$ of ferrule effect $[10,11]$.

Then again, the cost of getting this support in teeth with no coronal dentin is the additional loss of tooth tissue, and although a ferrule would be desireable, it should not be provided at the expense of the remaining coronal or root structural. However, it is important to bear in mind that a ferrule effect is just one part of the restored endodontically treated tooth that represents a complex system. The clinical performance of the entire complex is also affected by several other factors including the post and core material, luting agent, the overlying crown, and functional occlusal loads [12].

All porcelain crowns were used in this case to restore endodontically treated maxillary incisors. The prostheses are considered as an established alternative compared to metalporcelain. To match natural adjacent teeth, all porcelain crowns offer better potential than metal-porcelain ones. They offered more favourable aesthetics by miming naturally the optical properties of the teeth. Another more recent factor influencing the choice of ceramic materials is the pronounced cost for high precious metals as gold [2,13].

\section{CONCLUSION}

The success of a restoration is determined by retention, stability, aesthetic (especially anterior teeth) as well as biological aspects. This case showed that traumatic injury on maxillary incisors can be treated with combination of endodontic and periodontic treatment, not only to maintain the tooth as long as possible but also to restore the teeth function and the aesthetic aspect of the tooth in accordance with stomatognatic system.

\section{REFERENCES}

[1] E. Yaman Dosdogru, F. Nur Gorken, A. Pinar Erdem, E. Oztas, "Maxillary incisor trauma in patients with class II division 1 dental malocclusion: associated factors". J Istanbul Univ Fac Dent, vol. 1, no.51, 2017 
[2] H. Dalenda, A. Inez, K. Imen, B. Harzallah, C. Mounir, "Restoring a fractured central incisor: a challenge to overcome”, Biomed J Sci \& Tech Res, vol.4, no.1, 2017

[3] L. Mishra, A.S. Khan, M.M.A.C. Velo, S. Panda, A. Zavattini, F.A.P. Rizzante, H.I.A. Vega, S. Sauro, M. Lukomska-Szymanska, "Effect of Surface Treatments of Glass Fiber-Reinforced Post on Bond Strength to Root Dentine: A Systematic Review”, Materials, vol. 13, 2020

[4] K. Patil, N. Khaligbinerjad, N. El-Refai, K. Williams, A. Mickel, "The effect of crown lengthening on the outcome of endodontically treated posterior teeth: 10year survival analysis", JOE, vol.45, no.6, 2019.

[5] S.A. Patil, S. Kulkarni, S. Thakur, B. Naik, "Crown lengthening procedure following intentional endodontic therapy for correction of supra-erupted posterior teeth: case series with long-term follow-up", J Indian Soc Periodontol, vol.20, pp.103-8, 2016.

[6] M.R.T. Ardakani, F. Khalilian, Z. Nathegi, A. Esmaeilnejad, N. Janbakhsh, "A review of the crown lengthening surgery; the basic concepts", BJMMR, vol.13, no.3, pp.1-7, 2016.

[7] M. Jakubonyte, K. Cesaitis, J. Junevicius, "Influence of glass fibre post cementation depth on dental root fracture", Stomatologija, Baltic Dental and Maxillofacial Journal, vol.20, pp.43-8, 2018.

[8] J.R. Kina, P.H. Dos Santos, E.F.U. Kina, T.Y.U. Suzuki, P.L. Dos Santos, "Periodontal and prosthetic biologic considerations to restore biological width in posterior teeth", J Craniofac Surg, vol 22, no.5, pp.1913-6, 2011.

[9] R.B. Carlos, M.T. Nainan, S. Pradhan, R. Sharma, S. Benjamin, R. Rose, "Restoration of endodontically treated molars using all ceramic endocrowns" Case Report in Dentistry Hindawi Publishing Corporation, 2013, 210763.

[10] R.M. Patel, P. Baker, "Fuctional crown surgery in the aesthetic zone - periodontic and prosthodontic considerations", Dent Update, vol.42, no.1, pp.36-42, 2015.

[11] S. Jovanovski, J. Popovski, A. Dakskobler, L. Marion, P. Jevnikar, "The influence of crown ferrule on fracture resistance of endodontically treated maxillary central incisors", Balk J Dent Med, vo. 21, pp.44-49, 2017.

[12] J. Juloski, I. Radovic, C. Goracci, Z.R. Vulicevic, M. Ferrari, "Ferrule Effect: A Literature Review", JOE, vol.38, no.1, pp:11-18, 2012.

[13] A. Lahiwel, I. Kalghoum, B. Harzallah, M. Cherif, D. Hadyaoui, "All-ceramic versus metal-ceramic tooth supported single crowns with a minimum follow-up time of 3 years; survival and complications: a systematic literature review", J Dent Oral Disord Ther, vol.6, no.1, 2018. 